\title{
Direct Numerical Simulation of a Jet Issuing from Rectangular Nozzle by the Vortex in Cell Method
}

\author{
Tomomi Uchiyama ${ }^{1}$, Masahiro Kobayashi ${ }^{2}$, Shouichiro Iio ${ }^{3}$, Toshihiko Ikeda ${ }^{3}$ \\ ${ }^{1}$ EcoTopia Science Institute, Nagoya University, Nagoya, Japan \\ ${ }^{2}$ Graduate School of Information Science, Nagoya University, Nagoya, Japan \\ ${ }^{3}$ Faculty of Engineering, Shinshu University, Nagano, Japan \\ Email: uchiyama@is.nagoya-u.ac.jp
}

Received October 3, 2013; revised November 3, 2013; accepted November 10, 2013

Copyright (C) 2013 Tomomi Uchiyama et al. This is an open access article distributed under the Creative Commons Attribution License, which permits unrestricted use, distribution, and reproduction in any medium, provided the original work is properly cited.

\begin{abstract}
Direct numerical simulation of a jet issuing from a nozzle having a rectangular cross-section is conducted. The vortex in cell (VIC) method, of which computational accuracy was heightened by the authors in a prior study, is used for the DNS. The aspect ratio of the nozzle cross-section is 15 , and the Reynolds number based on the shorter side length of the nozzle exit is 6700. The turbulence statics, such as the mean velocity and the turbulence intensity, are favorably compared with the experimentally measured results. The behavior of the large-scale eddies as well as the development of the turbulent flow is also confirmed to agree with the measurement. These indicate that the authors' VIC method is successfully employed for the DNS of rectangular jet.
\end{abstract}

Keywords: Rectangular Jet; Vortex in Cell Method; DNS; Vortical Structure

\section{Introduction}

Vortex in cell (VIC) method [1] is one of the simulation methods for incompressible flows. It discretizes the vorticity field into vortex elements and computes the time evolution of the field by tracing the convection of each vortex element using the Lagrangian approach. The Lagrangian calculation markedly reduces numerical diffusion and also improves numerical stability. Thus, the VIC method is eminently suitable for direct numerical simulation (DNS) and large eddy simulation (LES) of turbulent flows, and various results have been reported. Cottet and Poncet [2] applied the VIC method for the wake simulation of a circular cylinder, and captured the streamwise vortices occurring behind the cylinder. Cocle et al. [3] analyzed the behavior of two vortex systems near a solid wall, and made clear the interaction between two counter-rotating vortices and the eddies induced in the vicinity of the wall. Chatelain et al. [4] simulated trailing edge vortices, and visualized the unsteady phenomena caused by disturbances. These studies are concerned with time-developing free shear flows. But the VIC method has not been applied to turbulent flows bounded by solid walls, which are closely related with the turbulent friction and heat transfer.
The authors [5] performed the DNS of a turbulent channel flow, which is a representative example of wall turbulent flows. When applying the existing VIC method, the oscillation of the flow increased with the progress of the computation, and eventually the computation collapsed. This was caused by the fact that the consistency among the discretized equations is not ensured. This was also because the solenoidal condition for the vorticity is not fully satisfied. To overcome such problems of the existing VIC method, the authors [5] proposed the improved VIC method. The authors [5] also applied the improved VIC method to the DNS of the turbulent channel flow. The DNS highlighted that the time evolution of the flow is fully computed and that the statistically steady turbulent flow is favorably obtained. It also demonstrated that the organized flow structures, such as streaks and streamwise vortices appearing in the near wall region, are successfully captured and that the turbulence statistics, such as the mean velocity and the Reynolds stress, agree well with the existing DNS results.

Jet is frequently observed in many engineering applications. As the jet is the representative of free shear flows governed by the convection of the eddies having various scales, the VIC method solving directly the vor- 
ticity field promises to be usefully applied to the simulation. But the VIC method has been scarcely used for the jet simulation.

The objective of this study is to search for the applicability of the authors' VIC method [5] to the DNS of jet flows. The DNS of a jet issuing from a nozzle having a rectangular cross-section is performed by the VIC method. The jet velocity field was experimentally investigated by Iio et al. [6]. The aspect ratio of the nozzle cross-section is 15, and the Reynolds number based on the shorter side length of the nozzle exit is 6700 . The DNS results, such as the mean velocity and the turbulence intensity, are favorably compared with the measured ones. The behavior of the large-scale eddies as well as the development of the turbulent flow is also confirmed to agree with the measurement. These indicate that the authors' VIC method is successfully employed for the DNS of rectangular jet.

\section{Vortex in Cell Method}

\subsection{Vorticity Equation and Orthogonal Decomposition of Velocity}

For an incompressible flow, the vorticity equation is given by

$$
\frac{\partial \boldsymbol{\omega}}{\partial t}+\nabla \cdot(\boldsymbol{\omega u})=\nabla \cdot(\boldsymbol{u} \boldsymbol{\omega})+v \nabla^{2} \boldsymbol{\omega}
$$

where $\boldsymbol{u}$ is the velocity and $\boldsymbol{\omega}(=\nabla \times \boldsymbol{u})$ is the vorticity.

According to the Helmholtz theorem, the velocity $\boldsymbol{u}$ is the sum of the curl of a vector potential $\psi$ and the gradient of a scalar potential $\phi$ :

$$
\boldsymbol{u}=\nabla \times \boldsymbol{\psi}+\nabla \phi
$$

when $\psi$ is postulated to be solenoidal or $\nabla \cdot \psi=0$, the curl of Equation (2) yields the vector Poisson equation for $\psi$ :

$$
\nabla^{2} \psi=-\omega
$$

Substituting Equation (2) into the continuity equation and rewriting the resultant equation, the Laplace equation for $\phi$ is obtained:

$$
\nabla^{2} \phi=0
$$

Once $\psi$ and $\phi$ have been computed from Equations (3) and (4) respectively, the velocity $\boldsymbol{u}$ is calculated from Equation (2). The vorticity $\omega$ in Equation (3) is estimated from Equation (1). The vortex in cell (VIC) method discretizes the vorticity field into vortex elements, and calculates the distribution of $\omega$ by tracing the convection of each vortex element.

It is postulated that the position vector and vorticity for the vortex element $p$ are $\boldsymbol{x}_{p}=\left(x_{p}, y_{p}, z_{p}\right)$ and $\boldsymbol{\omega}_{p}$, respectively. The Lagrangian form of the vorticity equa- tion, Equation (1), is written as [7]:

$$
\begin{aligned}
\frac{\mathrm{d} \boldsymbol{x}_{p}}{\mathrm{~d} t} & =\boldsymbol{u}\left(\boldsymbol{x}_{p}\right) \\
\frac{\mathrm{d} \boldsymbol{\omega}_{p}}{\mathrm{~d} t} & =\nabla \cdot\left(\boldsymbol{u}\left(\boldsymbol{x}_{p}\right) \omega\left(\boldsymbol{x}_{p}\right)\right)+v \nabla^{2} \boldsymbol{\omega}\left(\boldsymbol{x}_{p}\right)
\end{aligned}
$$

When the position and vorticity of a vortex element are known at time $t$, the values at $t+\Delta t$ are computed from the Lagrangian calculations of Equations (5) and (6). In the VIC method, the flow field is divided into computational grid cells to define $\psi, \phi$ and $\omega$ on the grids. If $\boldsymbol{\omega}$ is defined at a position $\boldsymbol{x}_{g}=\left(x_{g}, y_{g}, z_{g}\right)$, the vorticity $\boldsymbol{\omega}$ is assigned to $\boldsymbol{x}_{g}$, or a vortex element with vorticity $\boldsymbol{\omega}$ is redistributed onto $\boldsymbol{x}_{g}$

$$
\boldsymbol{\omega}=\sum_{p}^{N_{v}} \boldsymbol{\omega}_{p} W\left(\frac{x_{g}-x_{p}}{\Delta x}\right) W\left(\frac{y_{g}-y_{p}}{\Delta y}\right) W\left(\frac{z_{g}-z_{p}}{\Delta z}\right)
$$

where $\Delta x, \Delta y$ and $\Delta z$ are the grid widths, and $N_{v}$ is the number of vortex elements. For the redistribution function $W$, various forms are presented [7]. To suppress the numerical dissipation, a high-order scheme, which preserves the three first moments of the distribution, twice continuously differentiable and symmetric, is employed for $W$ :

$$
W(\varepsilon)=\left\{\begin{array}{cc}
1-2.5 \varepsilon^{2}+1.5|\varepsilon|^{3} & |\varepsilon|<1 \\
0.5(2-|\varepsilon|)^{2}(1-|\varepsilon|) & 1 \leq|\varepsilon| \leq 2 \\
0 & |\varepsilon|>2
\end{array}\right.
$$

Equation (8) was used for the simulations of time-developing free shear flows [2-4]. The DNS of a turbulent channel flow was also successfully performed with Equation (8) by the authors [5].

\subsection{Discretization by Staggered Grid}

For incompressible flow simulations, the MAC and SMAC methods solve the Poisson equation, which is derived from the equation for pressure gradient and the continuity equation. These methods employ a staggered grid to ensure consistency among the discretized equations, and to prevent the numerical oscillation of the solution. The staggered grid would appear to be indispensable for discretizing the Poisson equation for $\psi$ and the Laplace equation for $\phi$, which are derived in the VIC method. However, staggered grids are not readily accommodated in the existing VIC method.

The authors proposed a VIC method using a staggered grid in their prior study [5]. Figure 1 shows the arrangement of the variables in the grid. The scalar potential $\phi$ and velocity $\boldsymbol{u}$ are defined at the center and sides of a grid cell, respectively. The vorticity $\omega$ and the vector potential $\psi$ are defined on the edges. 


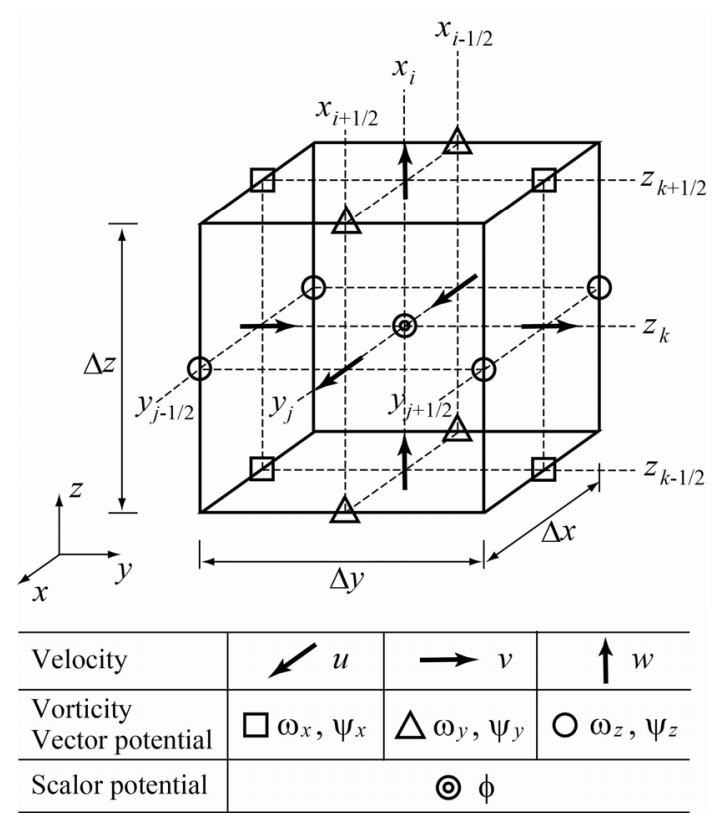

Figure 1. Staggered grid.

\subsection{Correction of Vorticity Field}

In the VIC method, the vorticity field is discretized into vortex elements, and the field is expressed by superimposing the vorticity distributions around each vortex element. The superposition is performed by Equation (7). The resulting vorticity field $\omega_{r}$ does not necessarily satisfy the solenoidal condition [7]. Denoting the vorticity satisfying this condition by $\boldsymbol{\omega}_{s}=(\nabla \times \boldsymbol{u}), \boldsymbol{\omega}_{r}$ is represented as [3]

$$
\omega_{r}=\nabla F+\omega_{s}
$$

where $F$ is a scalar function. Equation (9) corresponds to the Helmholtz decomposition of $\boldsymbol{\omega}_{r}$.

Taking the divergence of Equation (9), the Poisson equation for $F$ is obtained:

$$
\nabla^{2} F=\nabla \cdot \omega_{r}
$$

Calculating $F$ from Equation (10) and substituting into Equation (9) gives the recalculated vorticity $\omega_{s}$ [3]. This correction for vorticity needs to solve the Poisson equation, which increases the computational time. To reduce this additional cost, the authors [5] have proposed a simplified correction method.

The uncorrected vorticity, $\omega_{r}$, is linked to $\psi_{r}$ through Equation (3). Taking the divergence of Equation (3) and substituting Equation (10) into the resultant equation, the following relations are obtained:

$$
\begin{aligned}
\nabla^{2}\left(\nabla \cdot \boldsymbol{\psi}_{r}\right) & =-\nabla \cdot \boldsymbol{\omega}_{r} \\
& =-\nabla^{2} F
\end{aligned}
$$

Unlike the assumption for the solenoidal condition of $\psi$, the following equation for a non-solenoidal vorticity is derived from Equation (11).

$$
\nabla \cdot \boldsymbol{\psi}_{r}=-F
$$

Using $\boldsymbol{\omega}_{r}$ to calculate $\boldsymbol{\psi}_{r}$ from Equation (3), and determining $\phi_{r}$ from Equation (4), the curl of $\boldsymbol{u}_{r}$ transforms as follows:

$$
\begin{aligned}
\nabla \times \boldsymbol{u}_{r} & =\nabla \times\left(\nabla \phi_{r}+\nabla \times \boldsymbol{\psi}_{r}\right) \\
& =\nabla\left(\nabla \cdot \boldsymbol{\psi}_{r}\right)-\nabla^{2} \boldsymbol{\psi}_{r} \\
& =-\nabla F+\boldsymbol{\omega}_{r} \\
& =\boldsymbol{\omega}_{s}
\end{aligned}
$$

Equation (13) demonstrates that the curl of the velocity $\boldsymbol{u}_{r}$ calculated from $\boldsymbol{\omega}_{r}$ yields a vorticity $\boldsymbol{\omega}_{s}$ that satisfies the solenoidal condition. If the vorticity is recalculated by Equation (13), or the vorticity is corrected immediately after calculating the velocity by Equation (2), the discretization error in the vorticity is completely removed and the flow dynamics are accurately simulated without solving the Poisson equation, Equation (10). It should be noted that the staggered grid is required for rendering the transformation in Equation (13) applicable to the corresponding discretized equations.

\subsection{Simulation Procedure}

Given the flow at time $t$, the flow at $t+\Delta t$ is simulated by the following procedure:

1) Calculate the change in the strength of each vortex element defined on the grid, or calculate the vorticity $\omega_{p}$ from Equation (6).

2) Calculate the convection of each vortex element, or calculate the position $\boldsymbol{x}_{p}$ from Equation (5).

3) Redistribute the vortex element onto the grids, or calculate the vorticity $\omega$ on the grids by Equation (7).

4) Calculate the vector potential $\psi$ from Equation (3).

5) Calculate the scalar potential $\phi$ from Equation (4).

6) Calculate the velocity $\boldsymbol{u}$ from Equation (2).

7) Correct the vorticity, or calculate the corrected vorticity from the curl of $\boldsymbol{u}$.

\section{Simulation Conditions}

The DNS of an incompressible jet issuing from a nozzle having a rectangular cross-section is performed. The jet velocity field was experimentally investigated by lio et al. [6]. The computational domain is shown in Figure 2. The shorter side length of the nozzle exit is $w$, and the longer side length is $15 \mathrm{w}$. The $x$-axis is along the jet centerline, while the $y$ - and $z$-axes are parallel to the shorter and longer sides of the nozzle exit respectively. The computational domain consists of a cubic region of $40 w \times 40 w \times 40 w$. It is resolved into uniform grid cells of $400 \times 400 \times 400$. Consequently, the shorter and longer sides of the nozzle exit are divided by 10 and 150 cells 


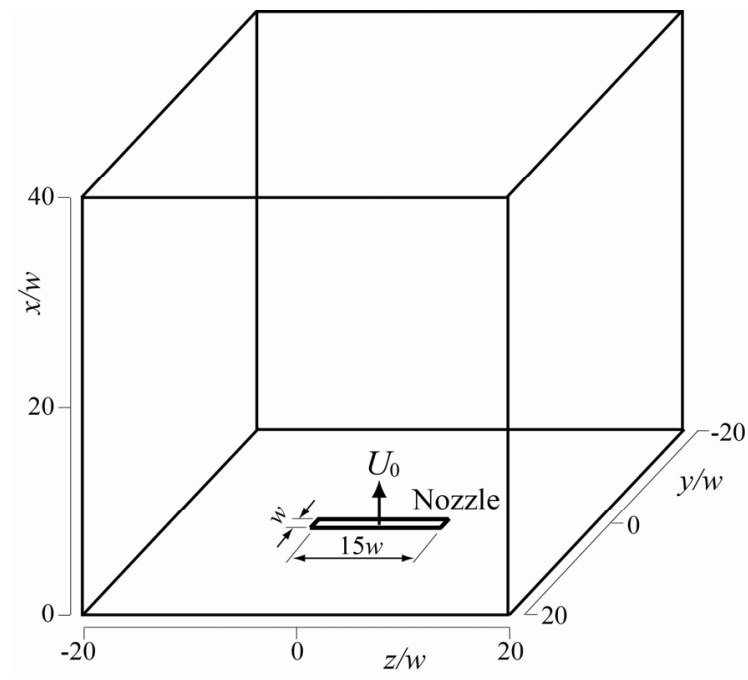

Figure 2. Rectangular nozzle and computational domain.

respectively. The jet velocity at the nozzle exit is $U_{0}$, and the Reynolds number based on $w$ and $U_{0}$ is 6700 . Table 1 summarizes the simulation conditions.

The boundary conditions are given as follows: Within the nozzle exit, a uniform and constant velocity $U_{0}$ is imposed.

$$
\begin{aligned}
& \left(u_{x}, u_{y}, u_{z}\right)=\left(U_{0}, 0,0\right) \\
& \partial \psi_{x} / \partial x=0, \psi_{y}=0, \psi_{z}=0 \\
& \partial \phi / \partial x=U_{0} \\
& \boldsymbol{\omega}=0
\end{aligned}
$$

The non-slip condition is adopted on the wall at $x=0$, on which the nozzle exit is mounted.

$$
\begin{aligned}
& \boldsymbol{u}=0 \\
& \partial \psi_{x} / \partial x=0, \psi_{y}=0, \psi_{z}=0 \\
& \partial \phi / \partial x=0 \\
& \omega_{x}=0, \omega_{y}=-\partial u_{z} / \partial x, \omega_{z}=\partial u_{y} / \partial x
\end{aligned}
$$

The velocity gradient is set at zero on the other boundaries. For example, the following conditions are imposed at $y= \pm 20 w$.

$$
\begin{aligned}
& \partial^{2} \phi / \partial y^{2}=0 \\
& \partial \psi_{y} / \partial x=0, \partial \psi_{z} / \partial x=0 \\
& \partial \psi_{x} / \partial x=-\partial \psi_{y} / \partial y-\partial \psi_{z} / \partial z
\end{aligned}
$$

The time increment $\Delta t$ is $w /\left(100 U_{0}\right)$. A fully developed flow was obtained at a time of $200 \mathrm{w} / U_{0}$. The time-averaged velocity and the turbulence intensity were acquired in the period from $250 w / U_{0}$ to $550 w / U_{0}$.

\section{Results and Discussion}

\subsection{Instantaneous Flow Fields}

The instantaneous distribution for the absolute value of
Table 1. Simulation conditions.

\begin{tabular}{cccc}
\hline Direction & $x$ & $y$ & $z$ \\
\hline Nozzle size & - & $w$ & $15 w$ \\
Domain length & $40 w$ & $40 w$ & $40 w$ \\
Grid cell number & 400 & 400 & 400 \\
Grid width & $w / 10$ & $w / 10$ & $w / 10$ \\
Nozzle outlet velocity & $U_{0}$ & 0 & 0 \\
Reynolds number $w U_{0} / \mathrm{v}$ & & 6700 & \\
Time increment $\Delta t$ & & $w /\left(100 U_{0}\right)$ \\
\hline
\end{tabular}

the velocity $|\boldsymbol{u}|$ for the fully developed jet is shown in Figure 3. The distribution on the central cross-sections of the jet is presented. Figure 3(a) shows the result on the $x-y$ cross-section at $z=0$ parallel to the shorter side of the nozzle exit. The potential core disappears at $x / w \doteqdot$ 2.6, as mentioned later. Thus, the jet spreads in the lateral direction downstream of the disappearing point, indicating the momentum diffusion in the direction. The distribution on the $x-z$ cross-section parallel to the longer side is shown in Figure 3(b). It should be noted that the jet width becomes slightly narrower as the streamwise distance increases.

The vorticity components $\omega_{z}$ and $\omega_{y}$ at the same instant as Figure 3 distribute as plotted in Figure 4, where the distributions on the jet central cross-sections are presented. The shear layers originate from the shorter and longer sides of the nozzle exit, and the turbulent flow rapidly develops just after the collapse of the shear layers.

Figure 5(a) shows the velocity on the jet central crosssection parallel to the shorter side of the nozzle exit, where the distribution near the nozzle is plotted. To make the distribution more understandable, the velocity $0.5 U_{0}$ is subtracted. On the shear layers originating from the longer sides of the nozzle exit, large-scale eddies occur at $x / w \geqq 2.2$. The eddies are non-axisymmetric around the jet centerline. They change into eddies having various scales as the streamwise distance increases. Figure 5(b) presents the flow field visualized experimentally by illuminating an alcohol mist with a laser light sheet [6]. The image clearly visualizes the occurrence of some largescale eddies on the shear layers as well as the rapid change of the flow into turbulence. It is confirmed that the DNS successfully resolves the flow development including the occurrence and collapse of large-scale eddies.

\subsection{Time-Averaged Velocity}

When the time-averaged velocity is calculated, the component in the jet axial direction $U$ distributes on the jet 


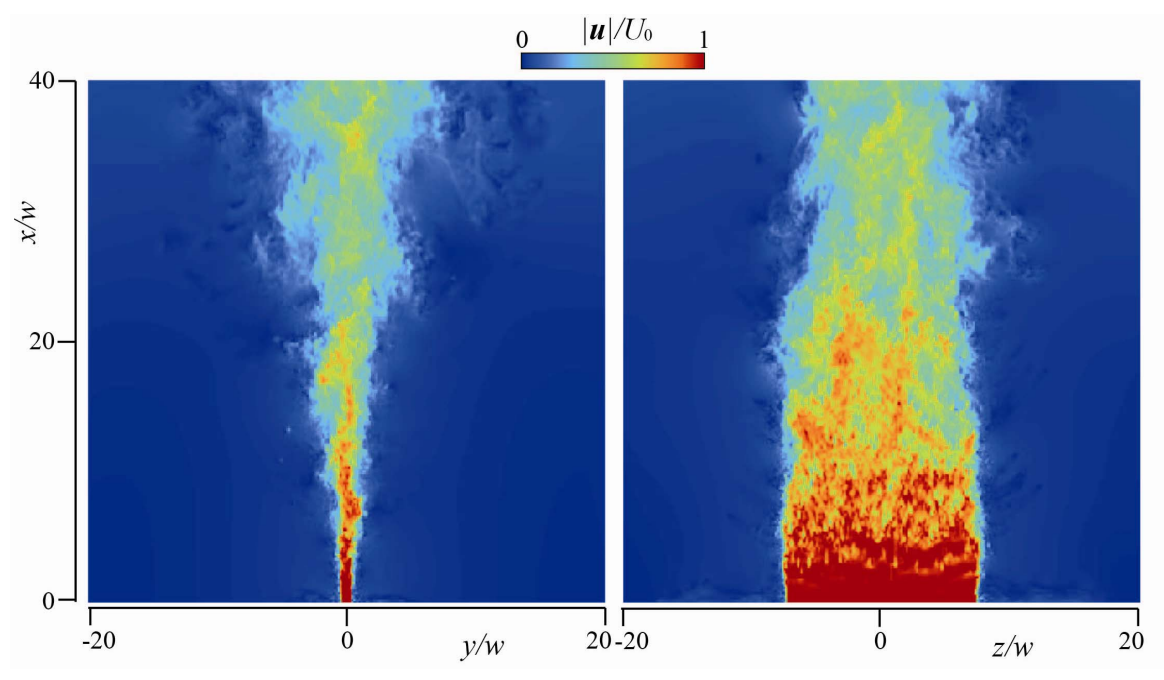

(a)

(b)

Figure 3. Instantaneous velocity distribution of fully-developed jet. (a) $x-y$ cross-section at $z=0$; (b) $x$ - $z$ cross-section at $y=0$.

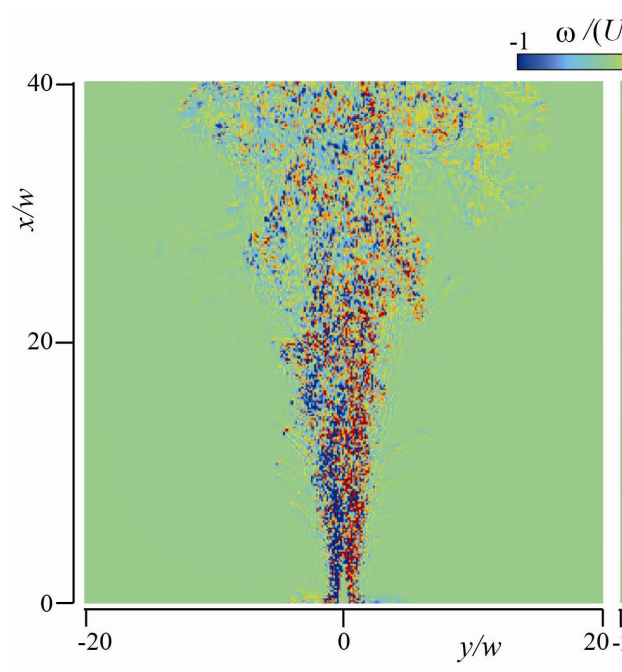

(a)

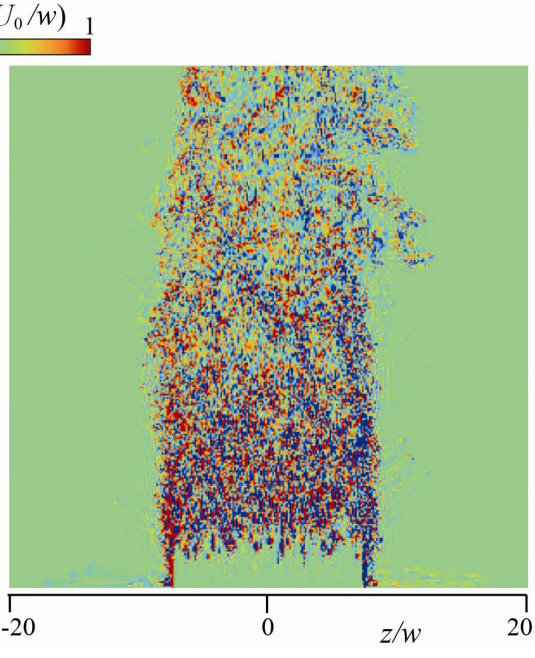

(b)

Figure 4. Instantaneous vorticity distribution of fully-developed jet. (a) $\omega_{z}$ on $x$-y cross-section at $z=0$; (b) $\omega_{y}$ on $x$ - $z$ cross-section at $y=0$.

central cross-sections as shown in Figure 6. On the $x-y$ cross-section parallel to the shorter side of the nozzle exit, the jet diffuses more in the lateral direction at $x / w \geqq 3$ as the streamwise distance increases. On the $x-z$ cross-section parallel to the longer side, however, the jet width becomes narrower at $x / w \geqq 3$ in the downstream direction.

Figure 7 compares the time-averaged velocity component $U$ obtained by the DNS with the measured result of Iio et al. [6]. A constant temperature hot-wire anemometer was employed for the measurement. It is found that the time-averaged flow features, such as the behavior of the potential core and the change in the jet width, are favorably grasped by the DNS.

The time-averaged velocity component $U$ distributes on the $y-z$ cross-sections as shown in Figure 8. The sections are parallel to the nozzle exit. Figure 8(a) presents the DNS result. With increasing streamwise $(x)$ distance, the jet spreads more in the $y$-direction. But it contracts gradually in the $z$-direction. These distributions agree well with the measurements depicted in Figure 8(b).

The time-averaged velocity on the jet centerline $U_{m}$ changes as a function of the distance from the nozzle as shown in Figure 9. The potential core exist until $x / w=$ 2.6, where the velocity decreases markedly. Comparing with the measured result [6], the disappearing point of the potential core locates slightly upstream, and the velocity decay is smaller. It is considered that the decay of the centerline velocity is markedly affected by the appearance and collapse of the large-scale eddies [8]. The 


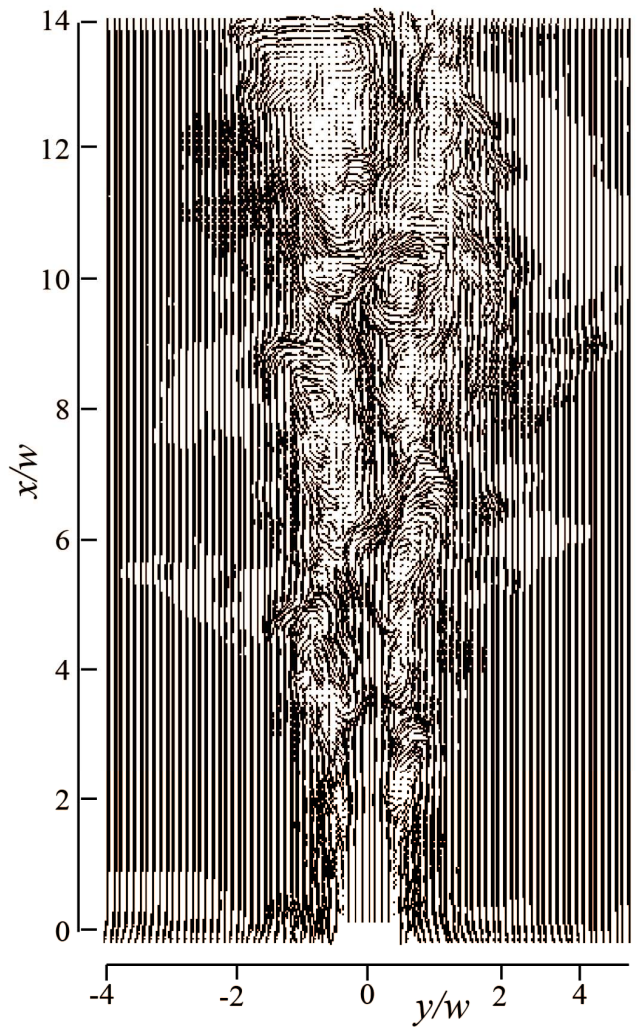

(a)

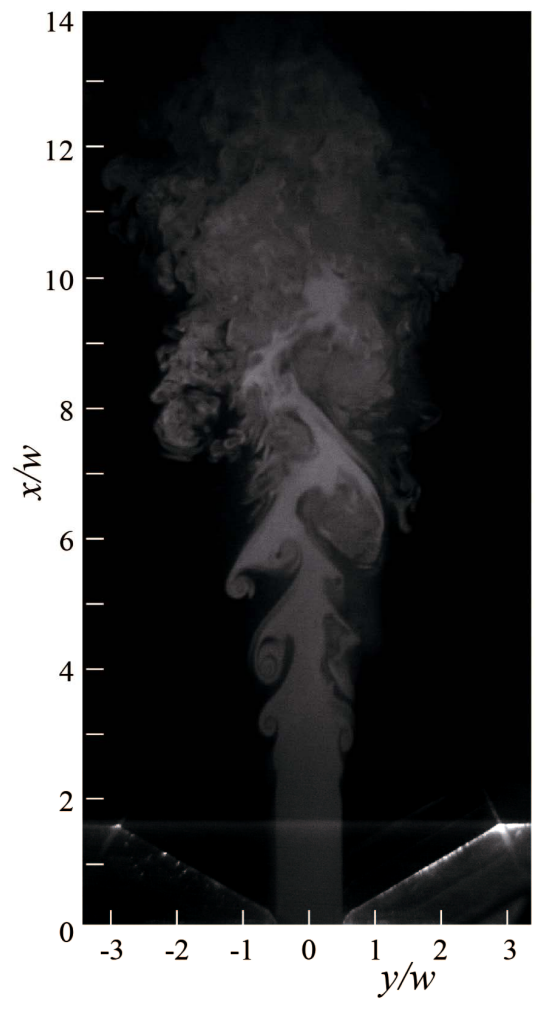

(b)

Figure 5. Flow field visualized on $x-y$ cross-section at $z=0$. (a) DNS; (b) Experiment.

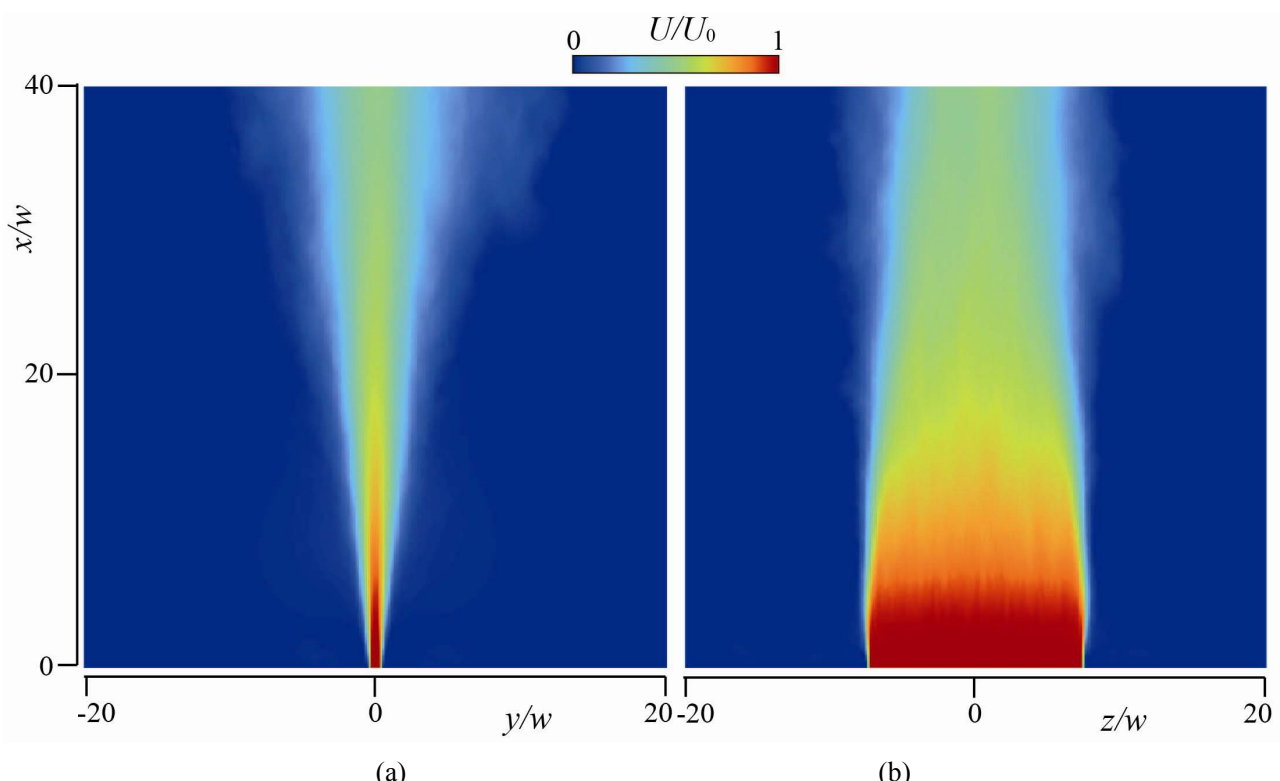

(a)

(b)

Figure 6. Distribution of time-averaged axial velocity. (a) $x-y$ cross-section at $z=0$; (b) $x-z$ cross-section at $y=0$.

behavior of the large-scale eddies depends on the velocity and turbulence intensity distributions within the nozzle exit. Some fluctuations exist in the velocity measured at the nozzle exit, and affect the velocity decay on the jet centerline through the occurrence and collapse of the large-scale eddies. The abovementioned discrepancy between the DNS and the measurement may be attributable to the fact that the boundary condition at the nozzle exit of the DNS does not accurately reproduce the velocity field of the corresponding experiments. 


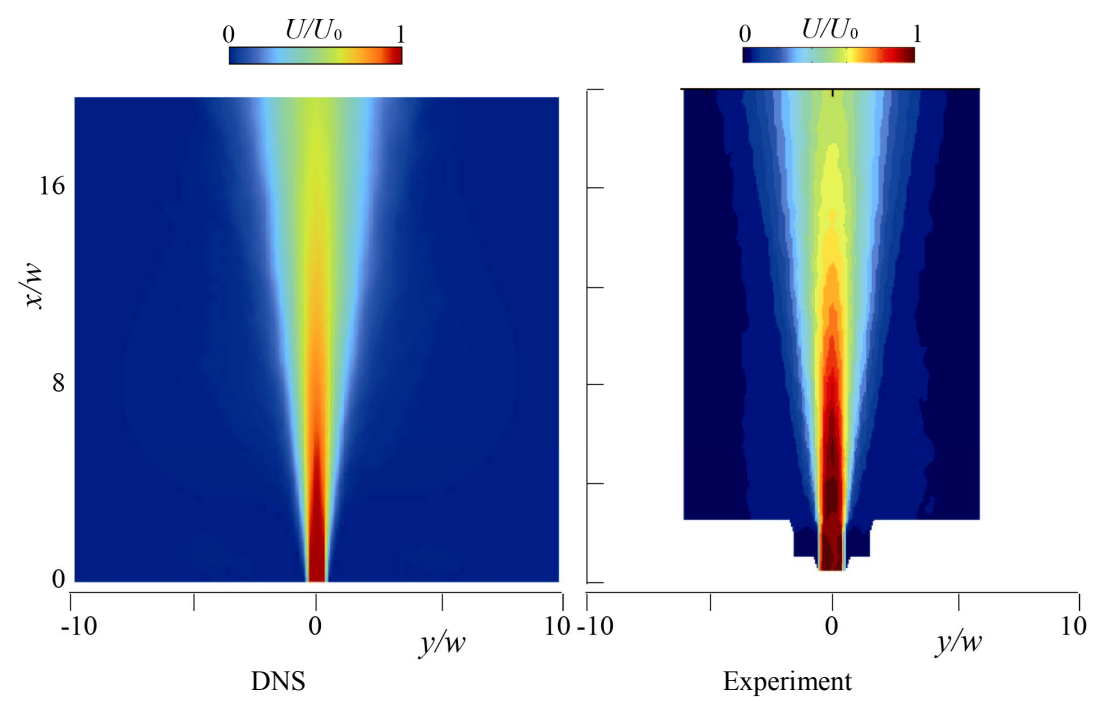

(a)

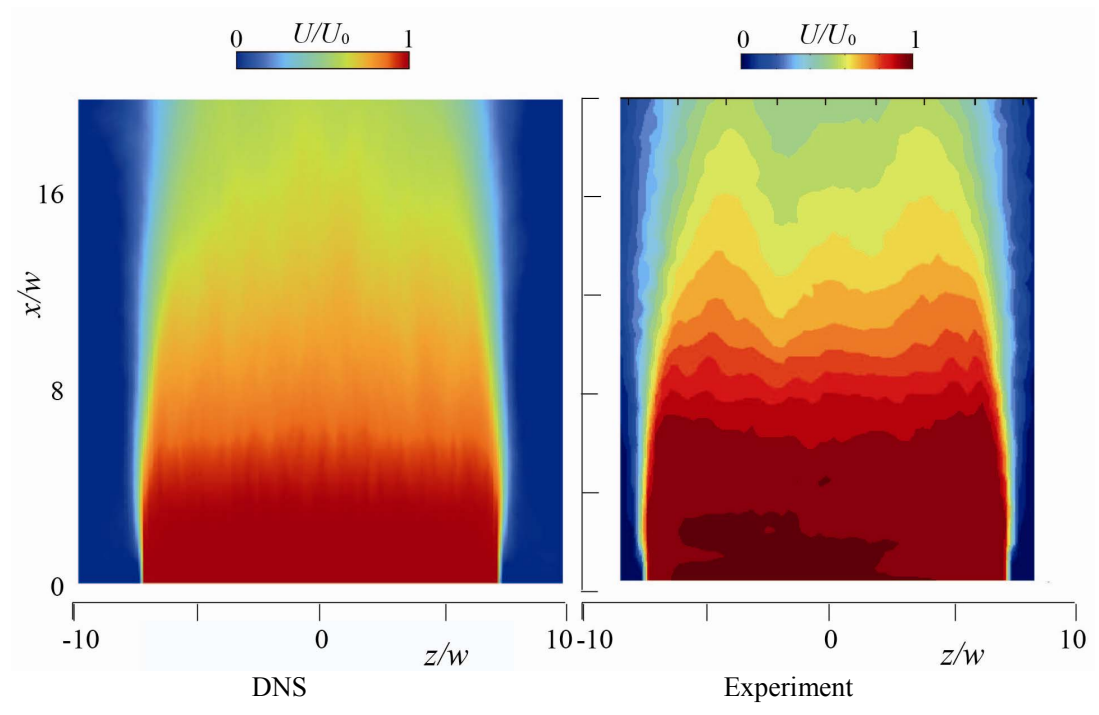

(b)

Figure 7. Comparison of time-averaged axial velocity near nozzle. (a) $x$-y cross-section at $z=0$; (b) $x-z$ cross-section at $y=0$.

The half widths along the shorter and longer sides of the nozzle exit, $b_{y}$ and $b_{z}$ respectively, change as shown in Figure 10. $b_{y}$ becomes larger as the streamwise distance increases. But $b_{z}$ reduces slightly. These DNS results agree almost with the measured ones [6].

\subsection{Turbulence Intensity}

Figure 11 presents the jet axial component of the turbulence intensity $u_{r m s}^{\prime}$. The distributions on the jet central cross-sections are plotted. On the $x-y$ cross-section parallel to the shorter side of the nozzle exit, $u_{r m s}^{\prime}$ is larger at $x / w \leqq 10$ on the shear layers originating from the longer sides of the nozzle exit. On the $x-z$ cross-section parallel to the longer side, $u_{r m s}^{\prime}$ takes the maximum value on the shear layers from the shorter sides.
In Figure 12, the turbulence intensity $u_{r m s}^{\prime}$ obtained by the DNS is compared with the measurement [6]. Though $u_{r m s}^{\prime}$ of the DNS is larger near the nozzle as well as on the shear layers originating from the shorter side of the nozzle exit, the trend of the distribution agrees well with the measurement.

The turbulence intensity on the jet centerline varies as shown in Figure 13. The turbulence intensity of the DNS hardly coincides with the measurement [6] near the nozzle $(x / w \leqq 4.2)$. This may be caused by the fact that the DNS ignores velocity fluctuations at the nozzle exit and the boundary layer on the nozzle wall, which exist in the corresponding experiment. But it should be noted that the turbulence intensity agrees well with the measurement at $x / w \geqq 4.2$ downstream of the disappearing point of the potential core. 

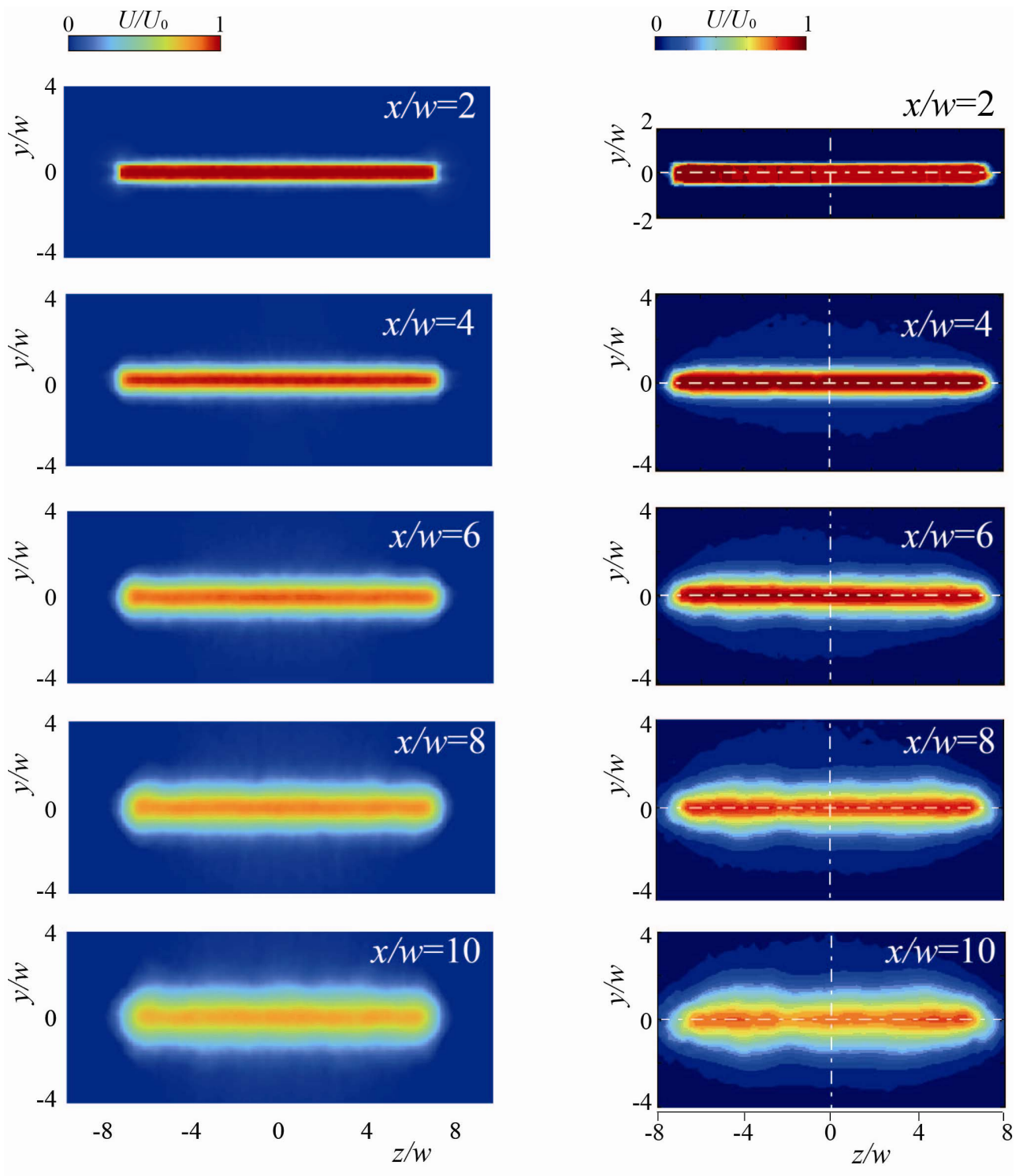

(a)

(b)

Figure 8. Comparison of time-averaged axial velocity on $y$-z cross-sections. (a) DNS; (b) Experiment.

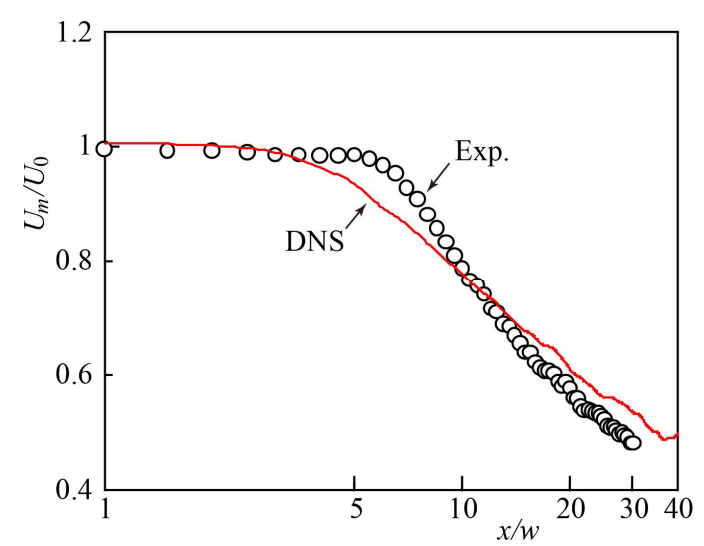

Figure 9. Axial evolution of jet centerline velocity.

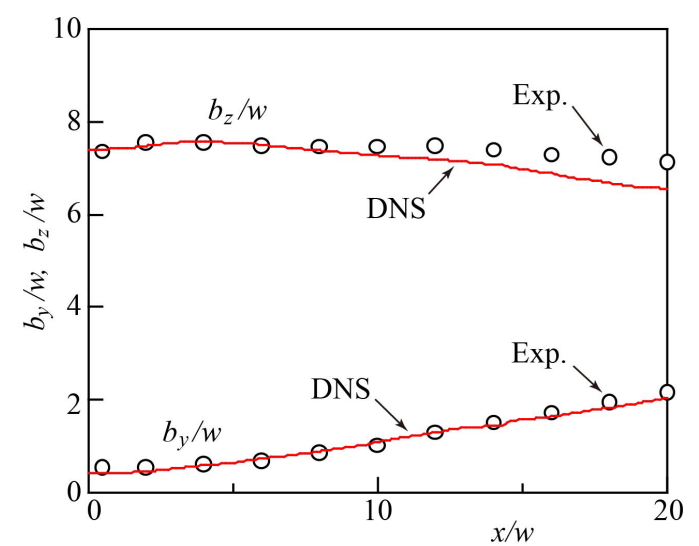

Figure 10. Axial evolution of jet half-width. 


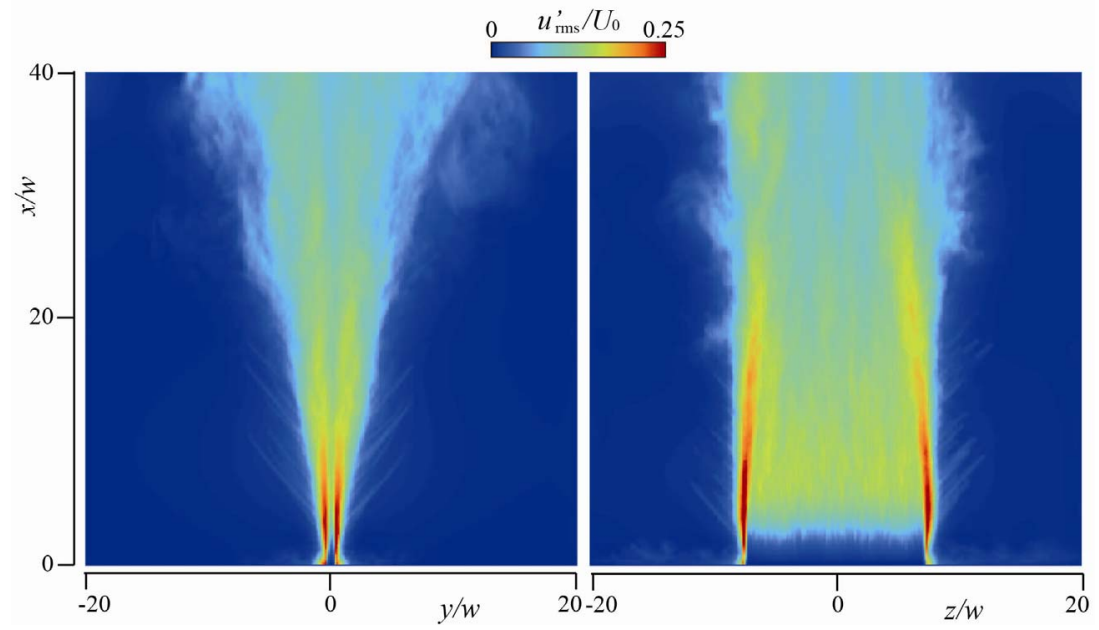

(a)

(b)

Figure 11. Distribution of axial turbulence intensity. (a) $x-y$ cross-section at $z=0$; (b) $x-z$ cross-section at $y=0$.

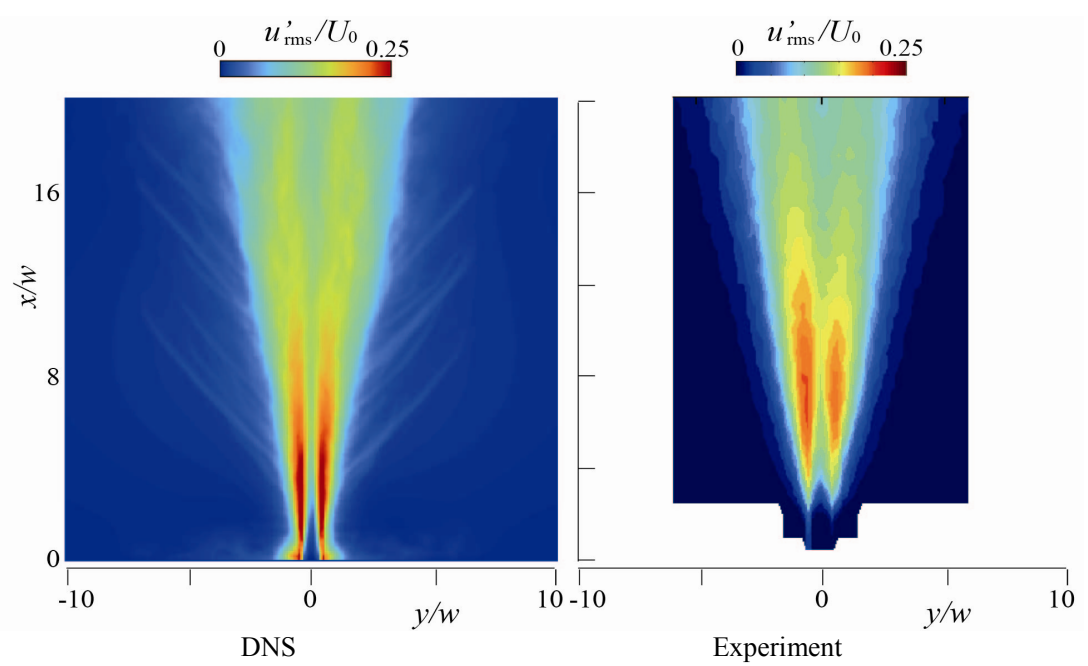

(a)

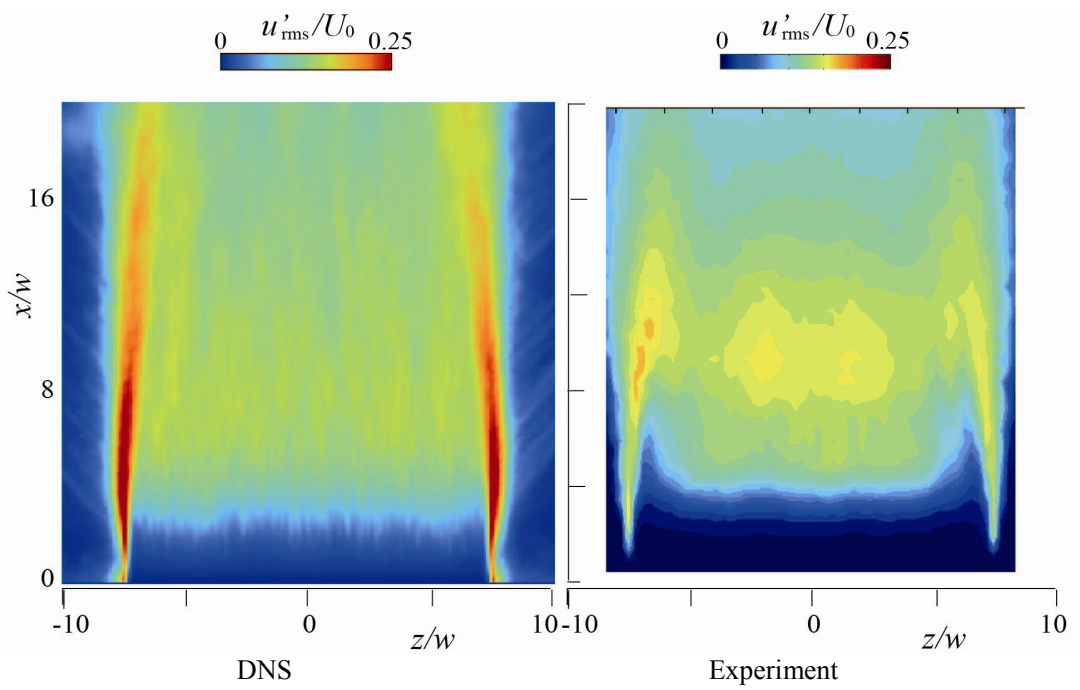

(b)

Figure 12. Comparison of axial turbulence intensity near nozzle. (a) $x-y$ cross-section at $z=0$; (b) $x$ - $z$ cross-section at $y=0$. 


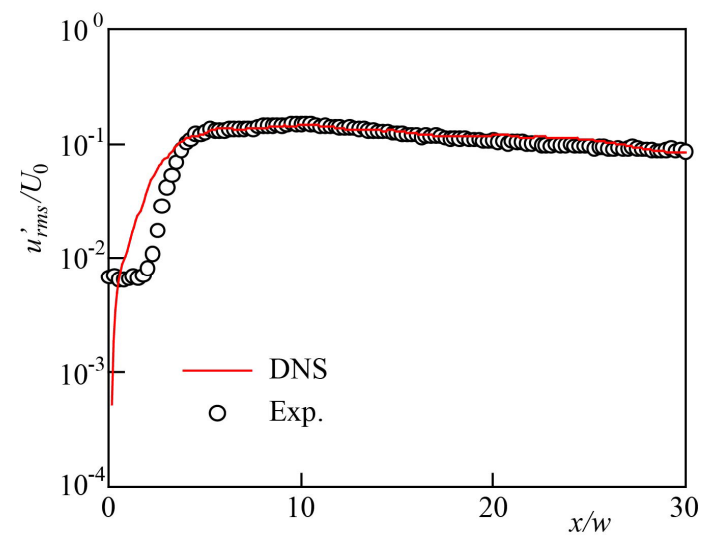

Figure 13. Axial evolution of axial turbulence intensity on jet centreline.

\section{Conclusions}

The DNS of a jet issuing from a nozzle having a rectangular cross-section was performed. The aspect ratio of the nozzle cross-section is 15 , and the Reynolds number based on the shorter side length of the nozzle exit is 6700 . The VIC method, of which computational accuracy has been heightened by the authors in a prior study, was used for the DNS. Staggered grids were employed to ensure consistency among the discretized equations, and to prevent numerical oscillations of the solution. A correction method of the vorticity was used so that the resultant vorticity field satisfies the solenoidal condition.

The DNS results, such as the mean velocity and the turbulence intensity, were favorably compared with the measured ones. The behavior of the large-scale eddies as well as the development of the turbulent flow was also confirmed to agree with the measurement. These indicated that the authors' VIC method is successfully employed for the DNS of rectangular jet.

\section{REFERENCES}

[1] I. P. Christiansen, "Numerical Simulation of Hydrody- namics by the Method of Point Vortices," Journal of Computational Physics, Vol. 13, No. 3, 1973, pp. 363379. http://dx.doi.org/10.1016/0021-9991(73)90042-9

[2] G.-H. Cottet and P. Poncet, "Advances in Direct Numerical Simulations of 3D Wall-Bounded Flows by Vortexin-Cell Methods," Journal of Computational Physics, Vol. 193, No. 1, 2003, pp. 136-158. http://dx.doi.org/10.1016/j.jcp.2003.08.025

[3] R. Cocle, G. Winckelmans and G. Daeninck, "Combining the Vortex-in-Cell and Parallel Fast Multipole Methods for Efficient Domain Decomposition Simulations," Journal of Computational Physics, Vol. 227, No. 21, 2008, pp. 9091-9120. http://dx.doi.org/10.1016/i.jcp.2007.10.010

[4] P. Chatelain, A. Curioni, M. Bergdorf, D. Rossinelli, W. Andreoni and P. Koumoutsakos, "Billion Vortex Particle Direct Numerical Simulations of Aircraft Wakes," Computer Methods in Applied Mechanics and Engineering, Vol. 197, No. 13, 2008, pp. 1296-1304. http://dx.doi.org/10.1016/j.cma.2007.11.016

[5] T. Uchiyama, Y. Yoshii and H. Hamada, "Direct Numerical Simulation of a Turbulent Channel Flow by an Improved Vortex in Cell Method," International Journal of Numerical Methods for Heat and Fluid Flow, 2013, in Press.

[6] S. Iio, K. Hirashita, Y. Katayama, Y. Haneda, T. Ikeda and T. Uchiyama, "Jet Flapping Control with Acoustic Excitation," Journal of Flow Control, Measurement \& Visualization, Vol. 1, No. 2, 2013, pp. 49-56. http://dx.doi.org/10.4236/jfemv.2013.12007

[7] G.-H. Cottet and P. D. Koumoutsakos, "Vortex Methods: Theory and Practice," Cambridge University Press, Cambridge, 2000. http://dx.doi.org/10.1017/CBO9780511526442

[8] S. Yuu, T. Nishioka and T. Umekage, "Direct Numerical Simulation of Three-Dimensional Navier Stokes Equations for a Slit Nozzle Free Jet and Experimental Verification," JSME International Journal, Series B, Vol. 36, No. 1, 1993, pp. 113-120.

http://dx.doi.org/10.1299/jsmeb.36.113 